“(C) 2021 IEEE. Personal use of this material is permitted. Permission from IEEE must be obtained for all other uses, in any current or future media, including reprinting/republishing this material for advertising or promotional purposes, creating new collective works, for resale or redistribution to servers or lists, or reuse of any copyrighted component of this work in other works." 


\section{An Individualized Countermeasure Assessment Framework for Astronauts in Space}

\author{
Jennifer Yeung \\ Ontario Tech University \\ 2000 Simcoe St North \\ Oshawa, Ontario, L1H 7K4 \\ jennifer.yeung@ontariotechu.net
}

\author{
Carolyn McGregor \\ Ontario Tech University \\ 2000 Simcoe St North \\ Oshawa, Ontario, L1H 7K4 \\ University of Technology Sydney \\ 15 Broadway \\ Ultimo, NSW 2007 \\ c.mcgregor@ieee.org
}

\section{INTRODUCTION}

Abstract - Preventive measures and countermeasure exercises play integral roles in maintaining the physiological health, wellbeing, and performance of astronauts in current day four to 11 month space missions to the ISS. While these activities help the body adapt to the adverse effects of spaceflight and minimize risks of physical deconditioning associated with weightlessness, these activities are inadequate for longer trips such as a 2-3 year mission to and from Mars. Furthermore, physical reconditioning and other issues such as visual impairment intracranial pressure (VIIP) syndrome remain for some astronauts returning from space missions. Combined with the effects of isolation and confinement in space, an astronaut's condition and performance can be compromised to a high degree with long-term impacts to their health and wellness upon returning to Earth if the appropriate interventions are not performed at appropriate times. Research has shown that representation of the relevant data to the active user during their activities has been proven effective in allowing them to perform the appropriate intervention to mitigate projected health risks. The physiological data and countermeasure equipment data currently located on the ISS has the potential to be correlated with the respective activities performed per astronaut for an individualized physiological monitoring approach for real-time health assessments. This paper presents a correlation method to enable individualized countermeasure assessments using big data collected during a simulated extreme environment workshop for firefighters as an analog. The online health analytics platform, created by McGregor, known as Artemis demonstrates this method using its capabilities in temporal abstraction for knowledge discovery, mechanisms for early detection of illnesses, and continuous real-time monitoring.

\section{TABLE OF CONTENTS}

1. INTRODUCTION ................................................. 1

2. BACKGROUND.................................................... 2

3. BIG DATA ANALYTICS FRAMEWORK FOR

COUNTERMEASURES IN SPACE................................ 3

4. Cold Stress Case STUdy.................................. 5

5. ANALOG FOR SPACE......................................... 6

6. CONCLUSIONS ....................................................8 8

ACKNOWLEDGEMENTS .......................................... 8

REFERENCES................................................8

BIOGRAPHY ..........................................................9
Space travel presents a high risk level to the physiological well-being of astronauts. The knowledge of space medicine and provisions for medical care in spaceflight has long been investigated by scientists and researchers with the purpose of extending space missions for longer durations. Aboard the International Space Station (ISS), astronauts follow countermeasure regimens based on the Russian system of preventive countermeasures that have been proven "sufficiently effective" from past missions [1]. These countermeasures allow astronauts to maintain their physiological condition and optimize their body's work performance throughout spaceflight. However, unique physiological changes occur daily, hourly, minute to minute, and even second-by-second. These changes occur in realtime, during countermeasure activities and during missionrelated tasks performed in space. Changes can also occur during periods of inactivity such as sleep or down-time. The individual training correction recommendations that are currently uploaded to the ISS weekly neglects the undetected unique physiological changes thereby missing data that can be integral in enabling real-time intervention to counter any potential long-term effects [1]. These changes can come as an adaptation cost, effecting the body's ability to adapt, deplete functional reserves, increase work tension, which in turn can affect their performance in conducting space mission-related tasks and ultimately deteriorate their health and physiological wellbeing upon their return to Earth [2].

To conduct the dense schedule of activities required per day on each mission, an astronaut's performance capacity must be optimal. Although astronauts perform countermeasure activities two hours per day to mitigate long-term health issues upon their return to Earth, the adverse effects of spaceflight still pose physical and mental deconditioning risks. Similar to astronauts in space, terrestrial community service workers such as firefighters are also exposed to physically and mentally demanding environments and mission-related tasks. Firefighter students at Durham College Pre-Service Firefighter, Education, and Training (PFET) program in Oshawa, Canada, are exposed to harsh environmental simulations as part of their training. Through the use of controlled climatic chambers at the Ontario Tech ACE Facility, they attend Extreme Environment Stress Workshops, where they are exposed to simulated 
environmental conditions pre-set to simulate extreme heat and extreme cold stress scenarios. The extreme environment scenarios conducted within this climate chamber facility serves as an analog environment for the firefighter students, presenting functional training scenarios to help students build adaption to field work; similar to analog missions that provide a terrestrial-based extreme space environment for astronauts in training, such as that of HERA, SIRIUS, and Antarctica mission experiments[3].

Approved by the ethics boards at Durham College and Ontario Tech University (approval \#156-1718 and \#14783), a study was conducted with firefighter students who wore the Hexoskin biometric compression shirt, a terrestrial companion product from the same organization, Carre Technologies Inc (Hexoskin), which produced the Astroskin Bio-Monitor now commissioned on the ISS. This enabled the collection of heart rate (HR), heart beat to beat (RR) interval data along with breathing rate and other metrics from 90 (19 female) participants from Durham College's PFET program. The research outlined in this paper is demonstrated using data from their Cold Stress training scenario. Physiological data is a form of big data. Big data analytics refers to the process of analysis on streaming data, such as data from physiological sensors and there is great potential for processing of physiological data to provide countermeasure assessment models in real-time [4].

Big data analytics was performed using McGregor's Big Data analytics platform, known as Athena. Athena is an online analytics platform that utilizes IBM's Infosphere Streams for the real-time analytics component and is capable of multiparticipant, multi-diagnosis, and multi-stream processing and analysis. Athena is an extension of the McGregor's Big Data analytics Artemis platform, which was originally designed to support neonatal populations in critical care units. The capabilities of Artemis to demonstrate subtle behaviors of early onset condition detections and adaptive health states have been presented in [4, 5]. Athena has been welldemonstrated to perform resilience assessment and development for the training assessments[7]-[11].

This paper presents a framework for an individualized countermeasure assessment method for astronauts in space that extends McGregor's prior big data frameworks. We use the firefighter case study as an analog to demonstrate that this extended framework of Artemis is capable of providing individual countermeasure assessments for astronauts in space. This analog characterizes the process that the extended framework supports for countermeasure feedback responses in real-time. The purpose of this paper is to conduct a demonstration of the capability of this framework that leverages the existing psychological data collected from firefighters who trained at the controlled climatic chambers in the Ontario Tech ACE Facility. An architecture enabling Artemis is demonstrated to ingest physiological data with exercise equipment data and training protocol data in realtime.
The next section presents a background of related work. Section 3 describes the extended online analytics framework which incorporates countermeasure data in space. Section 4 presents the case study scenario that relates the firefighter data in the context of individualized health assessment based on specific events. We then characterize the process this framework supports for countermeasure feedback responses in space in Section 5, with concluding remarks in Section 6.

\section{BACKGROUND}

Derived from studies in the early 1960s, heart rate variability analysis has been widely used in space medicine and clinical practice to assess the body's regulatory systems and provide information on functional health states[12]. A classification system derived using HRV analysis has been the subject of recent space medicine research. Bavesky et al developed a mathematical model at the Institute of Biomedical Problems (IBMP) at the Russian Academy of Sciences that identifies individualized functional health states of astronauts based on HRV [2]. The demonstration of that classification function based on HRV analysis in space medicine is performed on terrestrial studies and analog environments simulating microgravity. For participants in the Luna 2015 and Dry Immersion 2016 experiments, electrocardiogram (ECG) data was acquired and processed using a re-engineered functional health state algorithm within Artemis. Prysyazhnyuk et al were able to demonstrate the representation of dynamic health states over 5-minute, hourly, and daily time intervals $[4,12,13]$. The dynamic health states of participants with functional state data were calculated and presented for 5 minute segments and hourly, and more specifically, they demonstrated that HRV indices can show the degree and transition of functional health state trajectory of individual participants through the continuum of time within the online health analytics platform, Artemis [4, 13]. However, that work did not align the functional state calculations with activities or countermeasure exercises.

Artemis is a high frequency, multi-source health analytics platform with an architecture designed to improve health care and clinical decision support systems. Its robust online platform enables multi-patient, multi-diagnosis, and multistream temporal analysis for clinical applications in clinical management, medicine, and research. Developed and piloted for neonatal and pediatric intensive care units (NICU and PICU), Artemis is capable of handling high frequency data streaming, processing, persistency, and storage for simultaneous real-time monitoring and retrospective knowledge extraction. High speed physiological data from medical monitors including electrocardiogram (ECG), HR, respiration rate, and blood oxygen saturation $\left(\mathrm{SpO}_{2}\right)$ have been demonstrated in case studies implemented in NICUs in Canada, the USA, and China [14, 15]. Combined with electronic health record data provisioned with clinical proven algorithms, Artemis has been presented to advance diagnostics and prognostics for individualized patient monitoring providing a more holistic approach to real-time 
precision health care as a space medicine decision support system[17].

McGregor proposed a framework for the application of platforms such as Artemis to support autonomous health monitoring in space[18]. Yeung and McGregor in [19] introduced the extension of the online Artemis platform to incorporate countermeasure equipment data with physiological data to measure the efficacy of current countermeasure regimens. They proposed a framework in [20] that was instantiated within McGregor's Artemis platform to ingest physiological data and countermeasure data, perform analytics and processing in real-time on selected data, and to provide insightful information back to the astronaut with respect to their countermeasure activity enabling appropriate intervention or regimen change in realtime if necessary. To date, there has been no known studies that have demonstrated the observation of physiological responses correlating countermeasure equipment data and relevant activity within a continuous temporal window.

Countermeasure regimens are integral in every ISS mission. The current Russian system of preventive countermeasures that ISS crew members can follow, involve traditional exercises, interval workouts, and elevated heart rates for an average of two hours, twice a day, for three to four days per week[1, 20]. ECG, HR data, along with countermeasure equipment data (such as treadmill speed, force tension of bungees, cycle ergometer speed, and insole force from weightlifting) are currently amongst a myriad of data collected within specific experimental windows of time. There is great potential to provision the framework proposed in [20] with these categories of data, apply clinical algorithms, and advance current countermeasure systems to contribute to the domains of space health and medicine. More specifically, the demonstration of this framework would enable a health trajectory analysis of individual astronauts who have exercised in space, which can further provide knowledge and derive metrics to assess the efficacy of current countermeasure regimens for future longer duration space missions.

Similarly, for community support jobs such as firefighting, mental and physical challenges exist due the nature of tasks and environmental exposure of extreme conditions. Similar to astronauts, resilience training is therefore an important preventive activity to help firefighters ensure their safety, health, and wellness. As part of the Durham College PreService Firefighter, Education, and Training (PFET) program in Oshawa, Canada, firefighter students are required to attend an Extreme Environment Stress workshop, which consists of four simulated firefighting scenarios at the Ontario Tech University's ACE Facility climate chambers. One of the scenarios for training involves firefighters to ventilate a roof prop under cold stress conditions of $-20^{\circ} \mathrm{C}$ [8]. The purpose of this cold stress scenario is to simulate an environment for the firefighters similar to an event that could occur in Canadian winters.
These workshops occur annually for Durham College students and the students may elect to participate in research studies in collaboration with Ontario Tech University during their participation in each of these scenarios as part of the research ethics approved research study in 2018 and 2019, Students wore the Hexoskin biometric compression shirts during all of the four scenarios to enable the collection of physiological data [19]. With the data collected, RR intervals derived from the electrocardiogram were generated by the Hexoskin software and as noted previously, were synchronized with activities conducted in their training scenario for each individual participant and their standard deviation of N-N (SDNN) values were derived for each activity for each participant. Population based analysis were completed and the results were stratified based on gender. The results are discussed in [8]. For the SDNN values in the cold stress scenario in 2019, the results will be in a forthcoming publication elsewhere. For this paper, we demonstrate how the cold study scenario SDNN data from the firefighter students can demonstrate how exercise equipment used by firefighters can show their response to the bike ride exercise component of the training scenario and in so doing demonstrate how this is an analog for astronauts utilizing countermeasure devices. The next section will detail the components of the extended big data analytics framework of Artemis that enables the countermeasure feedback assessment representation for individual astronauts in space.

\section{Big Data Analytics Framework for COUNTERMEASURES IN SPACE}

A proposed framework for integrating data from countermeasure activities in space is presented in [20]. That framework is replicated here in Figure 1 with an extended capacity instanced on Earth with Mission Control, similar to the platform described in [18].

The design of this framework is based on the multidimensional online health analytics platform, Artemis, designed initially to support intensive care environments[6]. It enables the multi-streaming of continuous data feeds and enables real-time analytics to support health management and efficacy of countermeasure activities onboard a space station for long duration space missions. This framework consists of seven components each with specific functions: Data Collection, Data Acquisition, Data Buffering and Transmission, Data Transformation, Data Analytics, Information Exploration, and Data Storage. Data collection begins with the countermeasure activities performed and active utilization of any of the onboard countermeasure devices. Three categories of data are possible for collection, including physiological data, machine output data, and training protocol data [20]. As data is generated, the Data Acquisition component is capable of acquiring data streams from multiple devices on a continual basis. Wired and wireless acquisition methods are both possible. The Data Buffering and Transmission component enables both realtime and retrospective ingestion of the raw data acquired. The 




Figure 1 - Health Analytics Monitoring for Astronauts on the ISS with Countermeasure Data

Data Transformation component transforms this data and prepares data topics for consumption by components downstream. Modular algorithms process data topics in the Data Analytics component. Parallel processing can also be enabled by the adaptive real-time API, which allow communication between algorithms to consume the required data types as needed by their assigned tasks and designed functions[22]. Online graphs are generated by the Information Exploration component for data representation in real-time and/or retrospective analysis. The Data Storage component enables data persistency and storage, which remains critical for retroactive research and knowledge discovery. The function of these components are further described in detail in [20].

A key component of the extension of this big data analytics framework integrated with countermeasure data, is provisioning Earth-bound spaceflight surgeons and astronauts themselves with continual feedback of their individualized countermeasure's effect upon their health trajectories while inflight. Enabled by the Data Analytics, Information Exploration, and the Adaptive API components, the following sub-sections describe three sub-components within the extended Artemis framework to support real-time astronaut health monitoring and assessment. The functions of these sub-components are depicted in Figure 2.

\section{Real-time Monitoring and Alerting}

The capability of real-time monitoring of physiological and countermeasure data as it is generated, is provided in this subcomponent. Raw countermeasure data sources (e.g. Physiological, device/machine, training protocol), can be observed in real-time. Functional algorithms designed for specific performance reviews can also be enabled within this sub-component. The real-time streaming analytics API that was initially designed to process data tuples within data streams captured live from monitors plays a critical role in support of real-time streaming of respective data required for observation for astronauts and respective medical support and countermeasure training teams [22].

\section{Countermeasure Intervention Decision Support}

Data topics in the Data Analytics component can be processed by intervention support algorithms, which enables countermeasure feedback for the astronauts from this subcomponent. Data from countermeasure activities conducted can be processed in real-time to provide Countermeasure 
Intervention Decision Support. Individualized health trajectories can be observed immediately, providing opportunity for individual astronauts to perform any interventions as required. Real-time and retrospective information exploration for Earth-based medical support teams and training staff would also be enhanced with each astronaut's individual physiological and health performance trajectories.

\section{Visualization Reporting}

Countermeasure decision support is demonstrated in [20] based on the extended Artemis' multi-streaming, multidimensional analytics platform. The Visualization Reporting sub-component allows for individual astronaut performance reporting and assessment. Physiological data associated with respective events that occur while a countermeasure activity is performed can be represented via visual assessment reporting tool in this sub-component. Primed with algorithms such as the Functional Health State algorithm noted earlier, individual health trajectories can be presented.

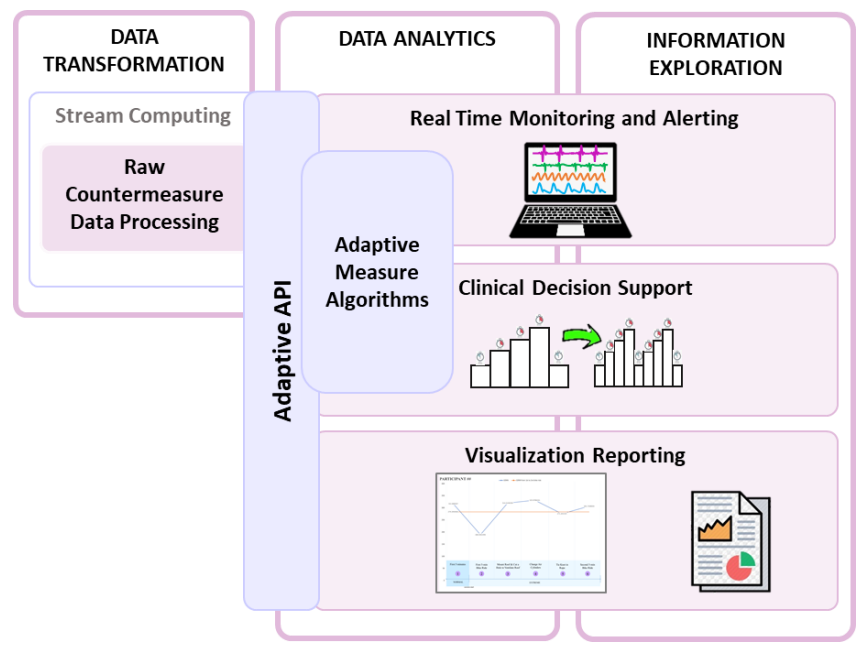

Figure 2 - Artemis Extended Framework for Real-time Feedback Based on Countermeasure Data

\section{Cold Stress Case Study}

A case study is presented in this section to demonstrate the ability of this framework to acquire measurements from countermeasure or intervention activities and to depict the physiological changes correlated to respective windows of activities in the Firefighters Cold Stress Scenario. The framework supports individualized assessments based on countermeasure events. We present the results of this case with visual assessments with data from the firefighter cold stress training scenario as an analog for this framework to apply for astronauts conducting countermeasures in space.

The purpose of the extreme cold scenario for the firefighter students is to simulate ventilating a roof in Canadian winters.
HR data and ECG values were collected using the Hexoskin garments for a total of 90 (19 female) individual participants. Cycle speed and equipment related data with respect to the individual monitored was also collected. Results are published elsewhere beyond the scope of this paper. Times of each participant having completed the entire scenario, including start and stop times of tasks were manually recorded on Microsoft Excel spreadsheets.

Firefighter students started the training scenario first by exercising on a stationary bike for five minutes to simulate the cardiac load of climbing onto the roof with their gear and in cold weather conditions. To simulate the precipitation, firefighters were sprayed with mist that froze as it landed, impacting their protective gear and equipment. They then climbed onto a reusable roof prop with an axe and were required to cut a hole for ventilation. Upon climbing back down from the prop roof, firefighters were also required to tie a specific knot in a rope as part of their fine motor skills exercise activity, followed by changing their air cylinders. Due to equipment and physical space limitations in the chambers, the rope-tie and change of air cylinders activities were conducted in different sequential order for some individuals. In addition, these activities are likely to occur for firefighters in the field whilst on a roof, however due to the space limitation on the prop roofs, firefighters were required to climb down first before performing the fine motor skills activities. Finally, to simulate an actual climb down a real roof in the winter, the firefighter students were required to exercise on the bike again for five minutes with their gear and the artificial precipitation sprayed on them before ending the scenario session.

From the ECG acquired for the duration of the Hexoskin garment worn by each firefighter, R peaks within the QRS complex and N-N intervals from the non-artifact R-R interval was performed in the Hexoskin cloud-based software. One file per participant was generated with derived HR values at one data point per second $(1 \mathrm{~Hz})$ along with a second file that contained R peak times in milliseconds. SDNN and mean HR of the first five minutes of wearing the Hexoskin as well as during the simulation scenarios and sub tasks were calculated for each participant. In addition, the minimum, maximum, and average HR data was also calculated. While the Artemis platform supports components for data collection, transmission, processing, storage and analysis of stored data, this paper focuses on Data Analytics and the Information Extraction components, as depicted in Figure 2, on the processed data to demonstrate an assessment framework for real-time monitoring of countermeasure exercises for the firefighters. The Data Collection, Transmission and Transformation had been performed previously on the data. The same framework will be discussed for functionality and capability to be used for astronauts in space in the next section.

With the physiological windows of data collected from the firefighters, the activities conducted respectively per participant during the cold stress scenario were annotated by 


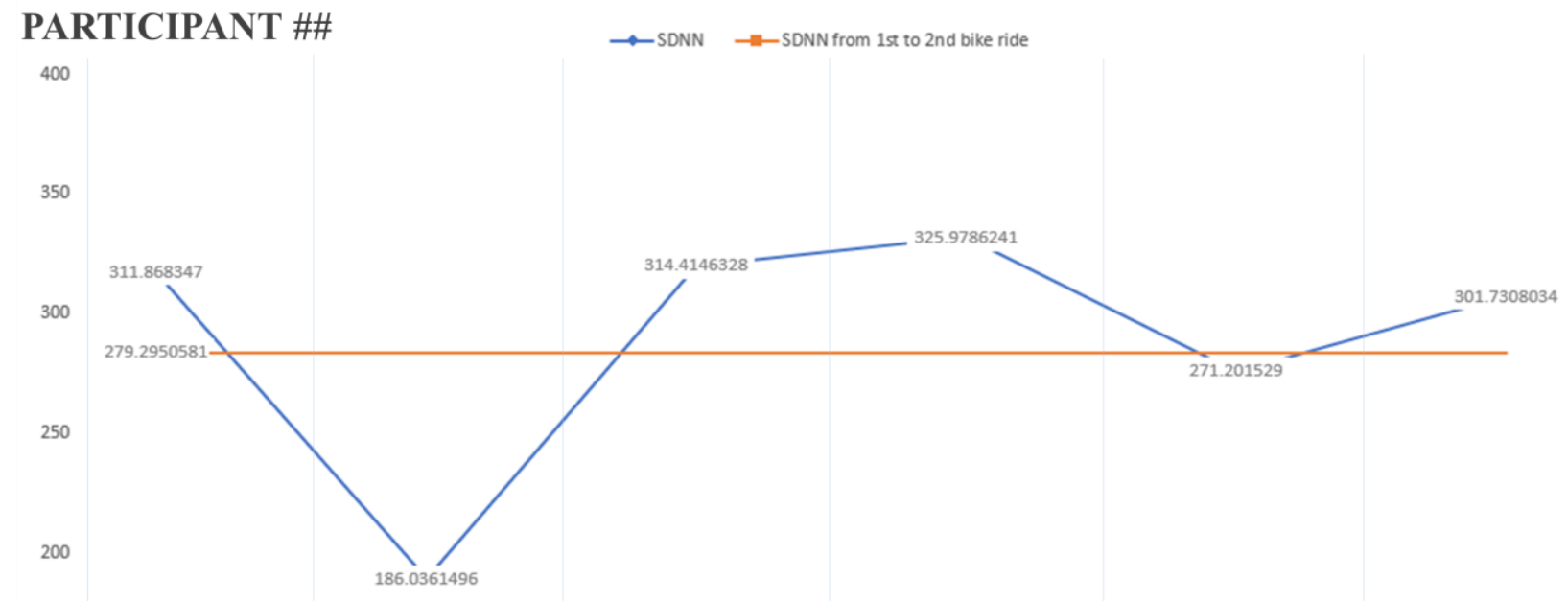

150

100

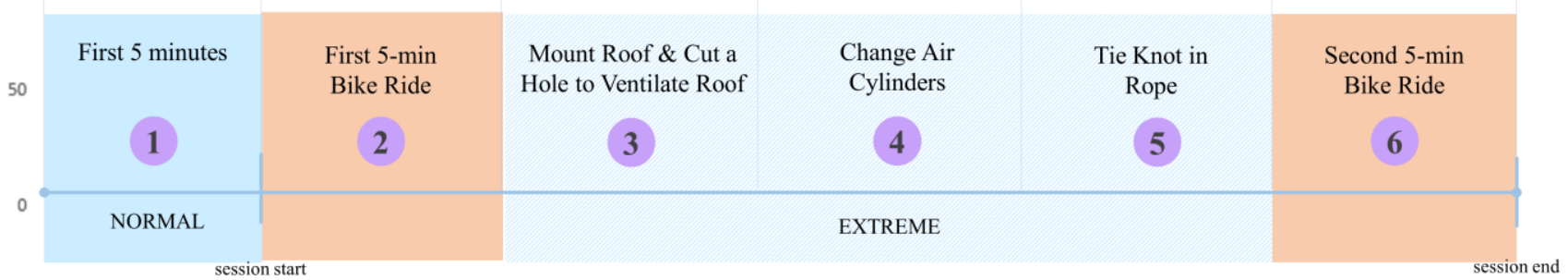

Figure 3 - Visual assessment report of analytics based on physiological data per activity performed by one participant in the Firefighter Cold Stress Training Scenario

research technicians and was later transferred into Microsoft Excel. The derived SDNN values per activity segment was then correlated to the sequential activities actioned. Depicted in Figure 3, the five-minute bike ride represents the first cardiac load event for a participant over a time continuum of activities conducted through the training workshop to the second five-minute bike ride which represents descending from a roof in the Winter. From this graph, the orange coloured windows of the bike rides represent the cardiac load event. The respective SDNN values represent the participant's SDNN trajectory. Since SDNN is derived from HRV, a reduced SDNN value in the second cardiac load event signifies a physiological stress load on the participant [23]. Additional information from the exercise equipment may also be ingested within this proposed framework to demonstrate the acquisition capacity of Artemis's online analytics architecture. Similar to terrestrial analog settings that provide simulation environments for astronauts-intraining to go to space, analytical systems simulated in Earthbased environments may also provide an analogous method to simulate its support for astronauts in space.

\section{ANAlog FOR SPACE}

Countermeasure training is integral in every mission, whether it is a firefighting mission or a mission in space. To advance current countermeasure systems with information that would enrich researchers and individual professional trainees and astronauts, the potential of real-time intervention is necessary. The extension of Artemis proposed in this paper would enable an alternate category of knowledge discovery with respect to the effectiveness of countermeasure exercises in preparation for, and even in, extreme environments. The case study described in the previous section demonstrates an extended health assessment architecture that incorporates countermeasure activities to enable real-time assessments and real-time intervention for firefighter resilience training.

For firefighters, the nature of their environment remains Earth-bound. Their exercise activity segment on the stationary bike aims to activate their cardiovascular system, elevating their HR before performing mission-related activities on the staged rooftop. Once they have completed their mission activities in the simulated (or real-life) scenarios, they were returned to a normal environment and their physiological adaption can be developed based on more experience and continued exposure to training. Their physiology from the extreme environment remains unchanged. The stress cases of the cold stress workshop can 


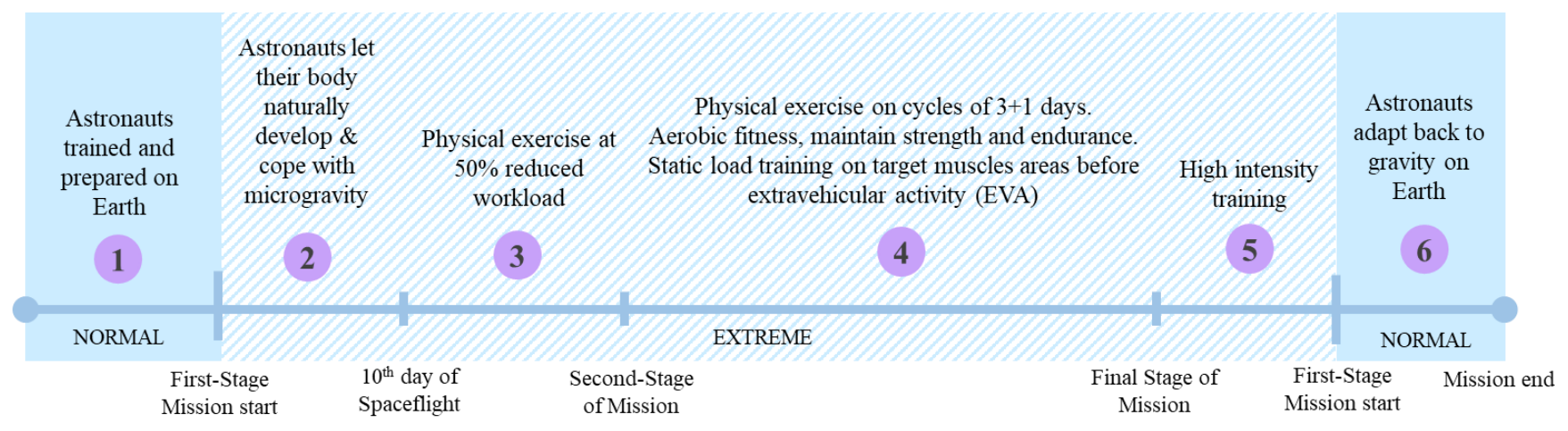

Figure 4 - Different stages of environment based on countermeasure sequence

be categorized into three phases tabulated in Table 1. We define environmental conditions under two categories: normal (ambient temperature) and extreme (extreme cold). Physiological stress levels are categorized by low, medium, and high. Physiological states are categorized under three states: steady (homeostasis), adaptive, and adaptive-steady (new homeostatic state where physiology has adapted). These classifications are used to demonstrate the parallel physiological states impacted by activities for firefighters and for astronauts.

Described in the section above, firefighters start the cold stress training scenario in a normal environment without exposure to the simulated cold weather conditions. Stress levels from their physiological perspective are relatively low and firefighters are at a steady (homeostatic) physiological state. When they enter the cold environmental conditions in the climatic chamber, firefighters must then adapt to the newly introduced extreme environment, therefore their physiological state must be adaptive. Furthermore, their exercise on the stationary bikes in the extreme cold weather conditions to simulate climbing up a roof presents physiological stressors on their body. From the visual reports depicting each participants' health assessment per activity, their SDNN values reflect the physiological state that they are in respectively. Their health trajectory with respect to the continuum of activities performed in this simulation environment enables a depiction of their adaptation abilities. Upon completing the training session, their physiological states at the point of their second bike ride in the extreme cold environment can be shown to be at an adaptive-steady state. This process can be observed in the individual health assessments presented in the sample visual in Figure 3 of the firefighters who participated in this study.

\section{Table 1. Classification of Stress Cases for Firefighter} Training

\begin{tabular}{|c|c|c|}
\hline Environment & $\begin{array}{c}\text { Physiological } \\
\text { Stress Level }\end{array}$ & Physiological State \\
\hline 1. Normal & Low & Steady \\
\hline 2. Extreme & High & Adaptive \\
\hline 3. Extreme & Medium & Adaptive-Steady \\
\hline
\end{tabular}

In the case for astronauts arriving at the ISS and living longterm in microgravity, changes to their physiology is inevitable. To mitigate this and to counter drastic impacts over time, consistent physical exercises in space play a critical role in their countermeasure activity. Astronauts must perform adaption interventions necessary to improve their health trajectory in real-time as their physiology adapts to the microgravitational environment. Astronauts exercise rigorously in space. One of the main countermeasure devices they use in space is the cycle ergometer (CEVIS), which is used as part of their effort to change their physiological trajectory to maintain their strength and optimal performance on ISS missions. For firefighters, simulation workshops including exercising on the bike for short periods is important for adaptation to extreme scenarios and resilience training. For astronauts, exercising on the bike is important for them to improve their long-term adaptation trajectories. Modelled similarly to the firefighters' cold stress scenario and referencing the mission events depicted in Figure 4, the cases for astronauts can be categorized into four phases tabulated in Table 2.

\section{Table 2. Classification of Stress Cases for Astronauts in} Space

\begin{tabular}{lcc}
\hline Environment & $\begin{array}{c}\text { Physiological } \\
\text { Stress Level }\end{array}$ & Physiological State \\
\hline 1. Normal & Low & Steady \\
\hline 2. Extreme & High & Adaptive \\
\hline 3. Extreme & Medium-Low & Adaptive \\
\hline 4. Normal & Medium & Adaptive-Steady \\
\hline
\end{tabular}

For astronauts, the second phase differ from the third phase largely based on acute adaption to microgravity in the first stage of a space mission to the ISS. We incorporate the consideration of time given for astronauts to adapt to the new environment in this phase. Physical exercises, which begin with a reduced workload of 50\% from the 5th to 10th day of spaceflight, are part of the routine countermeasures to help astronaut's physiological adaptation to microgravity [24]. 


\section{Conclusions}

Monitoring physiological health states and depicting performance assessments based physiological data collected from individuals conducting tasks in extreme environments for firefighters and for astronauts have been successfully demonstrated with Athena and Artemis.

This paper has presented a framework for individualized countermeasure assessment method for astronauts in space. It was instantiated within an extended Athena platform. That instantiation was demonstrated within a firefighter training scenario as an analog for spaceflight countermeasure activities.

Functional algorithms can be leveraged and analytics can run in real-time on high frequency data streamed to depict the dynamic health states of participants as their physiology reach homeostasis. The assessment of countermeasures for astronauts in real-time has been a neglected area of research. By extending Artemis to monitor individual SDNN values for firefighters during their 5-minute segments exercising on the bike, Artemis would also be able to ingest SDNN values from astronauts during their exercise segments on the CEVIS on the space station. The cold stress workshop scenario with the firefighters sufficiently served as an analog population study to demonstrate the health assessment framework presented in this paper to understand the physiological impact of countermeasures in extreme environments.

\section{ACKNOWLEDGEMENTS}

The authors wish to thank the Faculty from Durham College, the ACE Facility staff, together with all the Durham College students who participated in the study. They would also like to thank Michael Williams-Bell for his work with the instructors to design the scenarios in the ACE Facility. They would like to thank the research team who gathered the cold study data on the day that the workshops ran including Catherine Inibhunu, Rachit Desai and Brianna Grant.

This research is funded by McGregor's Research Excellence Chair in Health Informatics.

\section{REFERENCES}

[1] I. B. Kozlovskaya, E. N. Yarmanova, and E. V. Fomina, "The Russian system of preventive countermeasures: Its present and future," Hum. Physiol., vol. 41, no. 7, pp. 704-711, 2013.

[2] R. M. Baevsky, A. G. Chernikova, I. I. Funtova, and J. Tank, "Assessment of individual adaptation to microgravity during long term space flight based on stepwise discriminant analysis of heart rate variability parameters," Acta Astronaut., vol. 69, no. 11-12, pp. 1148-1152, 2011.
J. Perez, "Analog Missions," National Aeronautics and Space Administration, 2020. [Online]. Available: https://www.nasa.gov/analogs. [Accessed: 07-Jul-2020].

[4] C. McGregor, J. Heath, and Y. Choi, "Streaming Physiological Data: General Public Perceptions of Secondary Use and Application to Research in Neonatal Intensive Care," Stud. Health Technol. Inform., vol. 216, pp. 453-457, 2015.

[5] A. Prysyazhnyuk, C. Mcgregor, E. Bersenev, and A. V. Slonov, "Investigation of adaptation mechanisms during five-day dry immersion utilizing big-data analytics," 2018 IEEE Life Sci. Conf. LSC 2018, pp. 247-250, 2018.

[6] M. Blount et al., "Real-Time Analysis for Intensive Care," IEEE Eng. Med. Biol. Mag., vol. 29 (2), no. April, pp. 110-118, 2010.

C. McGregor and B. Bonnis, "Big data analytics for resilience assessment and development in tactical training serious games," Proc. - IEEE Symp. Comput. Med. Syst., vol. 2016-Augus, pp. 158-162, 2016.

C. McGregor, M. Williams-Bell, and B. Bonnis, "The use of Physiological Data for the Assessment of Firefighter Resilience,” Oshawa, 2020.

C. McGregor, B. Bonnis, B. Stanfield, and M. Stanfield, "Integrating Big Data analytics, virtual reality, and ARAIG to support resilience assessment and development in tactical training," 2017 IEEE 5th Int. Conf. Serious Games Appl. Heal. SeGAH 2017, pp. 1-7, 2017.

[10] M. Williams-Bell, C. McGregor, and B. Bonnis, "Heart Rate Responses of Pre-service Firefighters during Simulated Cold Environment Activities," 10th Annu. Mil. Veteran Heal. Res. Forum, pp. 4142, 2019.

[11] C. McGregor, F. M. Williams-Bell, and B. Bonnis, "Using Streaming Big Data for Resilience Assessment within Pre-service Firefighter Extreme Condition Training," 9th Annu. Mil. Veteran Heal. Res. Forum, p. 29, 2018.

[12] R. M. Baevskii, "Analysis of heart rate variability in space medicine," Hum. Physiol., vol. 28, no. 2, pp. 202-213, 2002.

[13] O. Orlov, C. McGregor, R. Baevsky, A. Chernikova, A. Prysyazhnyuk, and V. Rusanov, "Perspective Use of the Technologies for Big Data Analysis in Manned Space Flights on the International Space Station," 68th Int. Astronaut. Congr. (IAC 2017), 2017.

[14] A. Prysyazhnyuk et al., "Big data analytics for enhanced clinical decision support systems during 
spaceflight," 2017 IEEE Life Sci. Conf. LSC 2017, vol. 2018-Janua, pp. 296-299, 2017.

[15] C. McGregor, "Big Data in Neonatal Intensive Care," Computer (Long. Beach. Calif)., vol. 46, no. 6, pp. 54-59, 2013.

[16] C. McGregor, A. James, M. Eklund, D. Sow, M. Ebling, and M. Blount, "Real-time multidimensional temporal analysis of complex high volume physiological data streams in the neonatal intensive care unit," Stud. Health Technol. Inform., vol. 192, no. 1-2, pp. 362-366, 2013.

[17] C. McGregor, "A framework for online health analytics for advanced prognostics and health management of astronauts," Aerosp. Conf. 2015 IEEE, pp. 1-7, 2015.

[18] C. McGregor, "A platform for real-time online health analytics during spaceflight," IEEE Aerosp. Conf. Proc., 2013.

[19] J. Yeung and C. McGregor, "Countermeasure Data Integration within Autonomous Space Medicine: An Extension to Artemis in Space," in IEEE Life Sciences Conference, 2018, pp. 251-254.

[20] J. Yeung and C. McGregor, "Analyzing countermeasure effectiveness utilizing big data analytics for space medicine decision support: A case study," Proc. Int. Astronaut. Congr. IAC, vol. 2019Octob, no. October, pp. 21-25, 2019.

[21] I. B. Kozlovskaya and A. I. Grigoriev, "Russian system of countermeasures on board of the International Space Station (ISS): The first results," Acta Astronaut., vol. 55, no. 3-9, pp. 233-237, 2004.

[22] C. Inibhunu, R. Jalali, I. Doyle, A. Gates, J. Madill, and C. McGregor, "Adaptive API for Real-Time Streaming Analytics as a Service," 41st Int. Eng. Med. Biol. Conf., pp. 3472-3477, 2019.

[23] F. Shaffer and J. P. Ginsberg, "An Overview of Heart Rate Variability Metrics and Norms," Front. Public Heal., vol. 5, no. September, pp. 1-17, 2017.

[24] I. B. Kozlovskaya, A. I. Grigoriev, and V. I. Stepantzov, "Countermeasure of the negative effects of weightlessness on physical systems in long-term space flights," Acta Astronaut., vol. 36, no. 8-12, pp. 661-668, 1995.

\section{BIOGRAPHY}

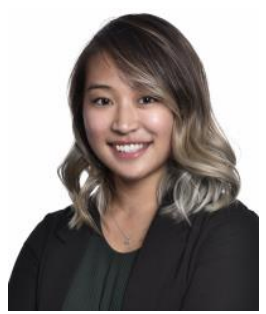

Jennifer Yeung holds a Bachelor of Aerospace Engineering degree, a Professional Master's Diploma in Aerospace Design Management, and is currently completing her Master of Computer Science degree at Ontario Tech University in Canada. Her research work focuses on space medicine decision support systems, which extends an existing online health analytics platform created for monitoring premature babies to monitor populations who undergo adaption training like firefighters and astronauts. Jennifer is a recipient of the Queen Elizabeth II Diamond Jubilee scholarship and she was the inaugural High Performance Computing Research Fellow at the Pawsey Supercomputing Centre in Western Australia. Jennifer is currently a research assistant in the Joint Research Centre in Artificial Intelligence for Health and Wellness between Ontario Tech and University of Technology Sydney. Prior to her current role, she was a sessional professor teaching aerospace and mechanical engineering in Toronto and she was an engineer in the Canadian aerospace industry for 5 years.

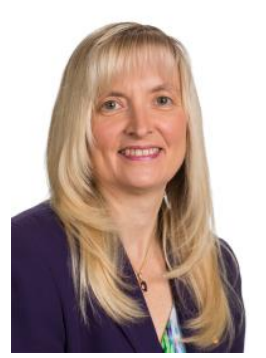

Professor Carolyn McGregor AM, PhD is the Research Chair in Artificial Intelligence for Health and Wellness and a two-time Canada Research Chair in Health Informatics at Ontario Tech University. She is the Founding Director of the Joint Research Centre in Artificial Intelligence for Health and Wellness between Ontario Tech University and University of Technology, Sydney. Dr McGregor AM has led pioneering research in Big Data analytics, artificial intelligence, deep learning, internet of things, temporal data mining and cloud computing. She now progresses this research within the context of critical care medicine, precision public health, mental health, astronaut health and military and civilian tactical training. 